\section{REAL TIME MONITORING OF SIMART GREENHOUSE ENVIRONMENT USING IOT FOR SUSTAINABLE AGRICULTURE}

KEY WORDS: IoT, Arduino

Uno, ESP32, WSN, Greenhouse effect

\section{Sadhana Tiwari*}

\section{Neha Sharma}

Ece" Prestige Institute Of Engineering Management \& Research, Indore" *Correspponding Author

Nowadays, the Wireless Sensors Network (WSN) and the Internet of Things (IoT) are widely used in providing decision support systems which solve many problems in the real-world. A greenhouse is a structure made mainly of transparent material, in which plants are grown in a controlled manner. Traditional method of monitoring greenhouse involves human labor and is very time consuming process. The proposed method deals with the scope of IoT based greenhouse technology in agriculture field for efficiently cultivating various crops irrespective of the geographical and environmental conditions. The IoT based greenhouse technology can provide control environment for high value crops like flowers, medicinal plants, etc. throughout the year to gain maximum profit. The greenhouse environmental parameters are continuously sensed using different parameters sensors and the collected data is displayed on a customized website.

\section{I.INTRODUCTION}

Agriculture has been practiced by human civilizations for a very long time. Various improvements were being made over the time and the greenhouse technology remained successful in ef ciently cultivating various crops irrespective of the geographical and environmental conditions. The traditional system for greenhouse monitoring is labor-intensive and time consuming. This proposed model provides solution for round the year and for all type of crop. It provides only controlled environment for the plants to prevent them from damage and thus increasing the overall produce. But IOT based smart greenhouse automatically controls the various parameters needed for the plants and sends the sensory data to a customized webpage for continuous and effective monitoring.

\section{Literature Survey}

Although India receives ample amount of precipitation and have many large river systems but still only one third of the total agricultural land is connected via canal irrigation system. Remaining majority of the portion is dependent on

monsoon or tube wells. Water collected on the surface also blocks pores in the soil and kills beneficial microorganisms. Places having limited supply of water cannot do irrigation throughout the growing season because the requirement of water often exceeds the supply due to conventional type of irrigation like sprinkler or in case allowing the water to just irrigate the field directly from water drainage channels. Excessive and irregular irrigation results in increase salinity, Water logging, hindrance in air communication to plant roots, reduction in temperature to soil, nitrate formation, increases acidity of soil.

Hence, problem lies in the mismanaged use of water. For optimum use of water, drip irrigation methodology can be adopted. Method targets the roots of plant to save water. Water obtained from all the sources like canal, rainwater harvesting, tube well etc. are not allowed to irrigate the fields directly; instead it is first stored into an underground tank. Tank is equipped with an ultrasonic sensor which measures the level of water continuously and alerts the user

with a SMS whenever water level falls below the threshold mark. Relative Humidity $(\mathrm{RH})$ affects leaf growth, photosynthesis, pollination rate and finally crop yield. Prolonged dry environment or high temperature can make the delicate sepals dry quickly and result in the death of flower before maturity. Hence it is very crucial to control air humidity and temperature. Temperature and humidity sensor placed inside the smart greenhouse to measure humidity and temperature. When temperature rises above a certain level, microcontroller will trigger relay attached to the fogger, which will sprinkle tiny water droplets of size of micron which will remain suspended in the air and bring the temperature down. In case the air moisture falls below the set value, similar mechanism will be triggered and the small water droplets will maintain the relative humidity $(\mathrm{RH})$.

Various wavelengths of light play a specific role for plant growth since different photosynthetic pigments within plants utilize different wavelengths. During morning, leaves receive it directly from sun but in order to boost up the rate of growth, we have provided the greenhouse with plant growing lights which will turn on whenever the reading from LDR sensor falls below cut-off value. Modern LED technology enable us to build LEDs that emit light in very specific spectra to achieve specific outcome in plant growth.

This effects primary and secondary metabolism, which are directly associated with the output quality. To avoid involvement of middleman and their adverse effects on farmers we proposed an IOT based solution, to monitor all the parameters and control the environmental conditions in the greenhouse accordingly.

\section{Proposed Model}

IOT based greenhouse system helps the farmers to carry out the work in a farm automatically without the use of much manual inspection. It protects the plants from extreme weather conditions namely: wind, hailstorm, ultraviolet radiations, and insect and pest attacks. Temperature,

humidity, light intensity and soil moisture are the parameter which is to be monitored in greenhouse. The data collected from the sensors are sending to the microcontroller for processing. The microcontroller is also connected to a Wi-Fi module (ESP-8266) which connects the system to the internet. After processing, the data is send over the Think to Speak (cloud) to be displayed on a customize webpage.

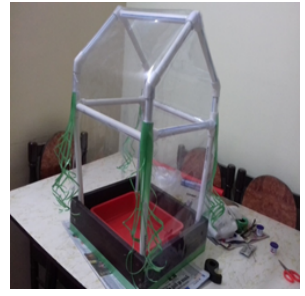

Fig 1. Proposed Model of Greenhouse system 
The greenhouse structure consists of a wooden plywood base which serves as the base. The body of the greenhouse consists of PVC pipes which are connected by $\mathrm{T}$ joints at the middle and $\mathrm{L}$ joints at the top to give an additional support to the greenhouse. The upper and middle pipe joints are connected with one another using thin steel wires to maintain the balance and durability of the structure. Further the whole pipe structure is attached to the plywood base with the help of the couplers which act as a supportive stand. The whole body of the greenhouse except the base is covered with plastic transparent sheet.

\section{System Design}

Proposed model consist of two modules: Hardware module and Software Module. Hardware Module requires ESP32, Arduino Uno, DHTl 1 Humidity and Temperature Sensor, Light Dependent Resistor, Soil Moisture Sensor, Humidifier and Dehumidifier, Relay and Power Supply

\section{ESP32:}

ESP32 is aWi-Fi-and-Bluetooth combo chip which operates on ISM $2.4 \mathrm{GHz}$ frequency band It is designed to get the best power and RF performance, showing different features like robustness, reliability and versatility ina varieties of applications. ESP32 is designed for mobile, wearable electronics, and Internet-of-Things (IoT) applications, mobile and wearable electronics devices. In a low-power IoT sensor hub application scenario, ESP32 is widely used. ESP32 is mainly used in Wi-Fi-and-Bluetooth IoT applications, with 20 external components. ESP32 integrates an antenna switch, power amplifier, low-noise receive amplifier, filters, and power management modules. Some Hardware details about ESP32-Wireless

- Integrated 802.11 BGNWi-Fi transceiver

- Integrated dual-mode Bluetooth (classic and BLE)

- WEP,WPA/WPA2 PSK/Enterprise

- Hardware accelerated encryption: AES/SHA2/Elliptical Curve Cryptography/RSA-409

- Onboard IPEX connector/PCB antenna for external antenna

\section{DHT 11 Humidity \& Temperature Sensor:}

The DHTll ultra-low-cost digital temperature and humidity sensor. It measures the surrounding air, and give digital signal on the data pin and there are no need analog input pins needed. For humidity sensing it uses two electrodes with moisture holding substrate between them.

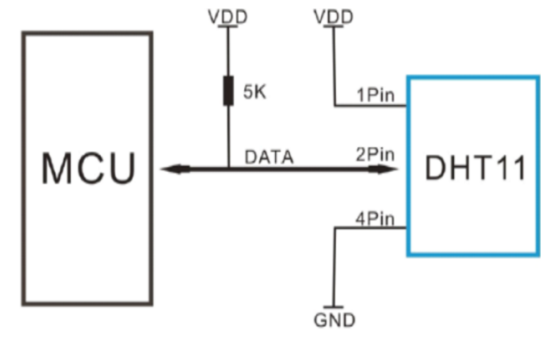

Fig 2. Interfacing of MCU with DHT Sensor

Interfacing

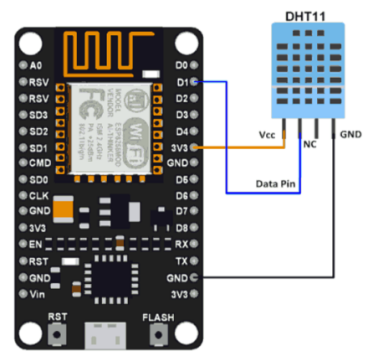

Fig 3.Interfacing of Node MCU with DHT sensor

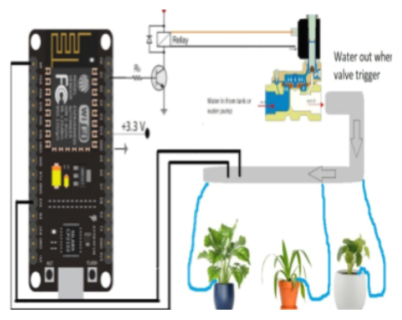

Fig 4 Interfacing of Node MCU with water Pump

\section{Light Dependent Resistor}

An LDR or light dependent resistor is also known as photo resistor, photocell, and photo-conductor. LDR is a resistor whose resistance depend on the amount of light falling on its surface. When the light incident on surface, then its resistance changes. A typical light dependent resistor has a resistance in the darkness of $1 \mathrm{MOhm}$, and in the brightness a resistance of a couple of Kohm.

\section{Humidifier and Dehumidifier:}

Thermo-electric dehumidifiers use thermoelectric effect to convert electricity into a temperature difference across a Peltier module. This create temperature difference. A thermoelectric dehumidifier is reliable and can be easily operate. It consists of-

- The Peltier module

- Two heat sinks - a smaller cold side heat sink and a larger (about twice as large as the cold side) hot side heat sink

- Afan and Relays

\section{Soil Moisture Sensor:}

Soil moisture sensors measure the volumetric water content in soil. The Soil Moisture Sensor uses capacitance to measure dielectric permittivity of the surrounding medium. The sensor creates a voltage proportional to the dielectric permittivity, and therefore the water content of the soil. Sensor can be connected in two modes; analog mode and digital mode. Soil Moisture Sensor has many applications, especially in agriculture.

\section{Arduino Platform:}

It is open source platform which is used to design electronics project. It is combination of hardware and a piece of software (Integrated Development Environment).

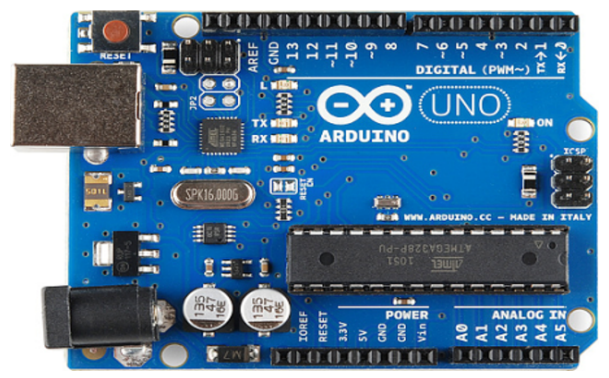

Fig 5. Arduino Uno Microcontroller

Thing Speaks Internet of things:

It is an open-source Internet of Things (IoT) application which is used to store and retrieve data from things using different protocol like HTTP and MQTT protocol over the Internet or via a Local Area Network. It is used to analyze and visualize uploaded data using MATLAB. It is used in different application like location tracking, social logging and sensor logging.

\section{V.Results}

Using Think speak, different parameters of greenhouse system like temperature, humidity, LDR, soil moisture are monitored in real time. The output window is given below: 


Field 1 Chart $40, x$

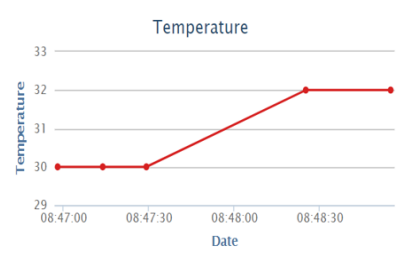

Fig 6.Temperature vs. time

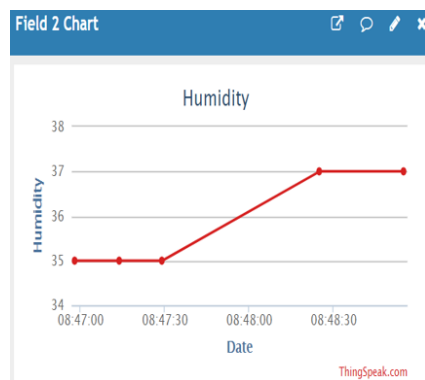

Fig 7 Humididty vs. time

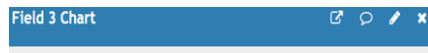

Soil Moisture

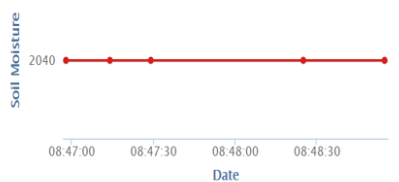

Fig 8. Soil Moisutre vs. time

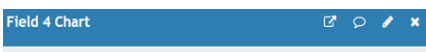

LDR

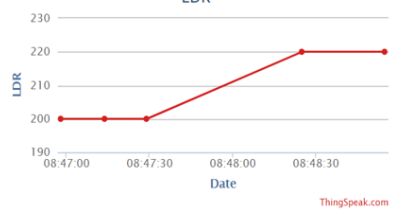

Fig 9. Soil Moisutre vs. time

\section{Applications}

Proposed model can be used in agriculture, botanical garden, Incubation centers for egg, Temperature dependent sex determination center for some reptiles. It can assist farmers in automating their crop production management and improving the quality of produce. This greenhouse can also be used for hatching and cultivation of eggs of some animal species as the temperature is continuously monitored and maintained inside the house.

\section{Conclusion}

The advantage of Smart Greenhouse over conventional farming is that we were able to produce insecticide and pesticide free crops and create a climate for the proper growth of plants and even provides alternative source of income through apiculture, incubation of eggs for some species of animals and temperature dependent sex determination of reptiles. Moreover, this system can be installed by any individual in his house (Rooftop greenhouse), who doesn't have knowledge about farming. it is possible to cultivate any type of crop using this controlled environment. Also all the necessary parameters such as humidity, soil moisture and light intensity are kept in mind according to the species of the plants. These parameters of all the sensors can be controlled by the user itself, making it much easier to cultivate various species and types of plants. The real time data of all the sensors with their digital values and characteristics can be monitored and controlled using Think Speak platform by the user. The automation also helps in reducing manpower and the errors which are the consequences of manually controlled farming. Thus, it also increases yield and rate of growth and produces organic agricultural products. It reduces human efforts and makes farming efficient and profitable activity.

\section{REFERENCES:}

1. Bseiso, B. Abele, S. Ferguson, P. Lusch, and K. Mehta, "A decisionsupport tool for greenhouse farmers in low-resource settings," in GlobalHumanitarian Technology Conference (GHTC), 2015 IEEE, Oct 2015,pp. 292-297.

2. K. A. Czyzyk, S. T. Bement, W. F. Dawson, and K. Mehta, "Quantifying water savings with greenhouse farming," in Global Humanitarian Technology Conference (GHTC), 2014 IEEE, Oct 2014,pp.325-332.

3. Z. Xiaoyan, Z. Xiangyang, D. Chen, C. Zhaohui, S. Shangming, and Z. Zhaohui, "The design and implementation of the greenhouse monitoring system based on gsm and rf technologies," in Computational Problem-solving (ICCP), 2013 International Conference on, Oct 2013,pp.32-35.

4. M. Mahdavian, M. B. Poudeh, and N. Wattanapongsakorn, "Greenhouse lighting optimization for tomato cultivation considering realtime pricing (rtp) of electricity in the smart grid," in Electrical Engineering/Electronics, Computer, Telecommunications and InformationTechnology (ECTI-CON), 2013 10th International Conference on, May 2013, pp. 1-6.

5. S. Kim, T. H. Shin, and J. S. Park, "A security framework in rfid multi-domain system," in Availability, Reliability and Security, 2007. ARES 2007. The Second International Conference on, April 2007,pp.1227-1234.

6. P. Laiolo, S. Gabellani, L. Pulvirenti, G. Boni, R. Rudari, F. Delogu,F.Silvestro, L. Campo, F. Fascetti, N. Pierdicca, R. Crapolicchio,S. Hasenauer, and S. Puca, "Validation of remote sensing soil moisture products with a distributed continuous hydrological model," in 2014 IEEE Geoscience and Remote Sensing Symposium, July 2014,pp. 3319-3322.

7. V. Sorathia, Z. Laliwala, and S. Chaudhary, "Towards agricultural marketing reforms: Web services orchestration approach," in 2005 IEEE International Conference on Services Computing (SCC'05) Vol-1, vol. 1, July 2005, pp. 260-267 vol.1.

8. Arduino Uno Rev3. Available:https://www.arduino.cc

9. ESP8266 Overview. Available:www.espressif.com.

10. Ji-chun Zhao, Jun-feng Zhang, Yu Feng, Jian-xin Guo, "The Study and Application of the IOT Technology in Agriculture", Computer Science and Information Technology (ICCSIT), 20103 rd IEEE International Conference, 911 July 2010.

11. Cui Wenshun, Cui Shuo, Yuan Lizhe, Shang Jiancheng, "Design and implementation of sunlight greenhouse service platform based on IOT and cloud computing", 2nd International Conference on Measurement, Information and Control (ICMIC), August 2013,Vol.01,pp. 141-144. 